\title{
Full Discharges In Fermilab’s Electron Cooler
}

\author{
L. R. Prost and A. Shemyakin \\ Fermi National Accelerator Laboratory, P.O. Box 500, Batavia, IL 60510, USA
}

\begin{abstract}
Fermilab's 4.3 MeV electron cooler is based on an electrostatic accelerator, which generates a DC electron beam in an energy recovery mode. Effective cooling of the antiprotons in the Recycler requires that the beam remains stable for hours. While short beam interruptions do not deteriorate the performance of the Recycler ring, the beam may provoke full discharges in the accelerator, which significantly affect the duty factor of the machine as well as the reliability of various components. Although cooling of $8 \mathrm{GeV}$ antiprotons has been successfully achieved, full discharges still occur in the current setup. The paper describes factors leading to full discharges and ways to prevent them.
\end{abstract}

Keywords: Discharge, electron, cooling, Pelletron, conditioning, protection, beam loss.

PACS: 29.17.+w; 41.75.Fr; 52.59.Mv; 52.80.Vp

\section{INTRODUCTION}

Recent demonstration of electron cooling of $8 \mathrm{GeV}$ antiprotons in the Recycler ring [1] became possible, in part, due to the stable performance of the electron beam generator [2]. The beam is generated in a Van-De-Graaff type electrostatic accelerator, Pelletron [3], which operates in a so-called energy recovery (or recirculation) scheme [4]. In this case the term means that the electron beam returns to the high voltage terminal, where it is dumped in the collector at the electron energy of $3 \mathrm{keV}$. The relative value of the current lost in the beam line is normally low, $\sim 10^{-5}$, and the protection circuitry closes the gun if the terminal voltage decreases because of higher losses. After these so-called "recirculation interruptions", the beam current is restored in $20 \mathrm{~s}$. Because the typical cooling time of antiprotons is $30-40 \mathrm{mn}$, the interruptions only result in a slight decrease of the average electron beam current. However, sometimes the Pelletron terminal discharges in a microsecond to a nearly zero voltage. These full discharges dramatically increase the pressure in the tubes, decrease the tube electric strength, and may damage the Pelletron electronics. The recovery time may take from $5 \mathrm{mn}$ for a simple check of the electronics and a modest pressure improvement, to a several hours long reconditioning of the tubes, to days to repair the electronics. A significant amount of efforts was put into understanding the mechanisms triggering the full discharges and into decreasing their frequency.

\section{TUBE ELECTRIC STRENGTH AND CONDITIONING}

Without beam, the discharge frequency depends on the magnitude of the electric field applied to a tube and on the tube history. If the high voltage applied to an 
acceleration tube is increased, at some level the tube pressure starts jumping. The jumps indicate discharges of single gaps of the tube and do not necessarily result in an immediate full discharge. However, the probability of a full discharge increases very rapidly above this point Below in the text we will refer to this level of the potential gradient along the tube as the tube's electric strength, $E_{t s}$.

Freshly installed acceleration tubes had tube strengths of $9 \mathrm{kV} / \mathrm{cm}$, which is well below the nominal gradient, $12 \mathrm{kV} / \mathrm{cm}$. The strength is significantly increased by conditioning. In our case, conditioning means a slow increase of the voltage applied only to one of six $60-\mathrm{cm}$ sections forming the Pelletron tubes. The voltage increase results in partial discharges, which improve the tube strength. Full discharges still occur, but because the stored energy at a given voltage gradient is much lower than for all sections together, they are much less harmful. Namely, when conditioning a single 60 -cm section, only $~ 5 \%$ of all full discharges deteriorate the tube strength; for two sections, it increases to $10 \%$; and, in attempts to condition all sections at once, the electric strength dropped for $\sim 50 \%$ of all full discharges.

Typically, conditioning of a single section is easy up to an electric field $E$ of $18 \mathrm{kV} / \mathrm{cm}$ but further increases are much slower. Usually, we stop the process when the electric field stays above $19 \mathrm{kV} / \mathrm{cm}$ for 5 minutes. After such conditioning the terminal voltage $U_{t}$ can be raised to $5.2 \mathrm{MV}(E=14.4 \mathrm{kV} / \mathrm{cm})$ before the first vacuum bursts are observed.

\section{FULL DISCHARGES WITH A BEAM}

The simplest explanation of why the frequency of the full discharges increases with the beam current is in the re-distribution of the electric field along the tube caused by beam losses. All tube electrodes are connected to a string of $0.48 \mathrm{GOhm}$ resistors. At the nominal voltage $U_{t}=4.32 \mathrm{MV}$, the string current is $39 \mu \mathrm{A}$, and a beam loss of only several $\mu \mathrm{A}$ can significantly change the electrode potentials. As a result, the electric field in some locations may approach $E_{t s}$, provoking a full discharge. Hence, to decrease the frequency of the full discharges, one needs to increase the tube strength beyond the working point as much as possible and minimize the beam loss to the tube electrodes.

The first recommendation was followed when the tube length was extended in the time of relocating the Pelletron from a test building [5] to its final location at the Recycler ring, thus decreasing the nominal electric field by $20 \%$. We feel that it made a dramatic effect on the reliability of operation.

There are several scenarios of how electrons can reach the tube electrodes. The most obvious one is when the primary beam touches an electrode because of errors in steering or focusing. Because the beam has sharp edges, scraping it off of any electrode by $\sim 0.1 \mathrm{~mm}$ results in a beam loss of $10 \mu \mathrm{A}$. Thus, every error of this type induces a full discharge. However, after the very first shifts, the beam position in the acceleration and deceleration tubes is well controlled by use of beam position monitors at the bottom of the tubes and by beam centering in the collector, such that these events are eliminated. Much more serious difficulties arise from primary beam loss in the time of a recirculation interruption and re-distribution of the potential in the deceleration tube caused by secondary electrons escaping the collector. 


\section{Beam in the acceleration tube}

If for some reason the beam is experiencing losses to the ground more than tens of $\mu \mathrm{A}$, both the gun voltage and the Pelletron potential decrease until the protection circuitry closes the gun. As a result, the beam trajectory and envelope are changing, and the primary electrons can reach the tube electrodes. The resulting full discharges in the acceleration tube were the major problem in the recirculation test [5], where it was overcome by the cut-and-try method.

The capacitance of the terminal to the ground is $300 \mathrm{pF}$, and the capacitance between the cathode and anode potential surfaces is $800 \mathrm{pF}$. A beam loss to the ground discharges both these capacitances, but the relative changes in the anode voltage $U_{a}$ are much stronger than in the Pelletron voltage. As a result, in the acceleration tube, where the beam trajectory is straight, the main effect is the changes of the beam envelope caused by the dropping anode voltage.

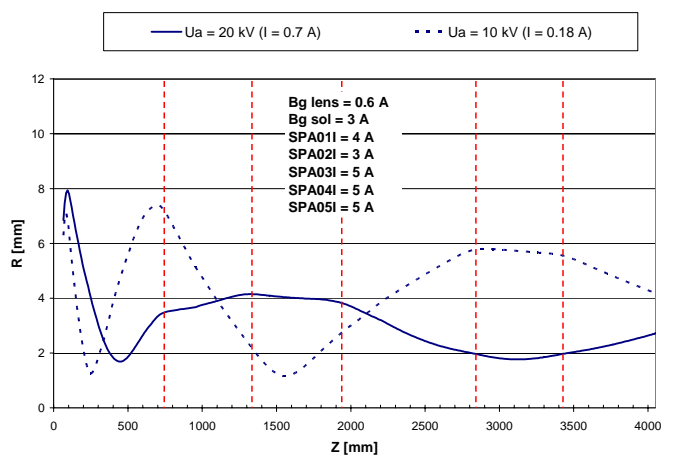

(a)

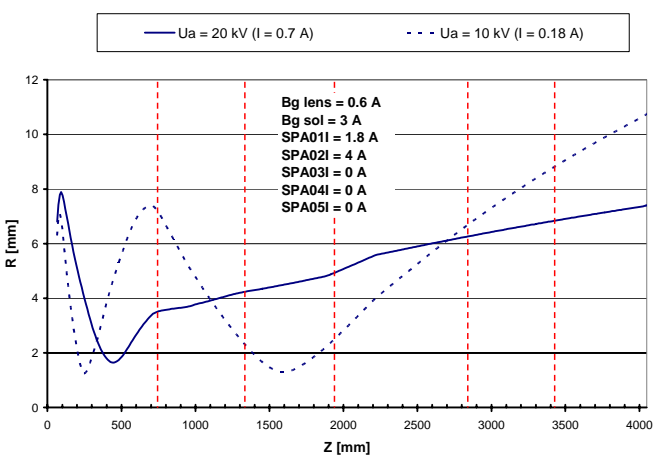

(b)

FIGURE 1. Simulations of the beam envelope in the accelerating column at the nominal anode and terminal voltages (solid curve) and at the time of recirculation interruption (dotted curve). (a) Nominal focusing settings; (b) alternate focusing settings. The limiting aperture is $12 \mathrm{~mm}$. Vertical dotted lines represent the approximate locations of the magnetic lenses.

To prevent this type of discharges, the beam envelope in the acceleration tube was simulated with the SAM/SuperSAM/BEAM software package $[6,7,8]$ before beginning the work in the final setup. First, a set of focusing lens currents which allowed the beam transport at nominal parameters $\left(U_{t}=4.32 \mathrm{MV}\right.$, beam current $I_{b}=0.7 \mathrm{~A}, U_{a}=20 \mathrm{kV}$ ). was found. Then, the simulation was repeated for lower anode voltages with all other parameters fixed, modeling the beam behavior in the time of interruptions. The maximum beam envelope radii were typically found at $U_{a} \sim 10 \mathrm{kV}$ $\left(I_{b}=0.18 \mathrm{~A}\right)$. Finally focusing settings were adjusted to pass the beam far from the aperture at this intermediate voltage, and the process was repeated.

The final result and one of the intermediate steps are shown in Fig. 1. This work allowed operating with no full discharges in the acceleration tube.

\section{Protection of the deceleration tube}

We still do not know the optical properties of the entire beam line accurately enough to reliably predict the beam envelope in the deceleration tube. Hence, the procedure described in the previous section is not applicable there. In addition, at 
present settings, the dispersion in the deceleration tube is comparatively large, $\sim 0.5 \mathrm{~m}$, and the beam shifts significantly when the Pelletron voltage decreases.

The first solution, which worked successfully in the recirculation test [5], is the use of a so called crash scraper. The scraper was kept close to the beam so that in the time of an interruption the disturbed electrons are lost at the scraper and not at the tube electrodes. However, for the concept to work, the beam size modifications in the time of an interruption and the dispersion at the location of the crash scraper should be significantly larger than the corresponding values in the deceleration tube, which imposes inconvenient restrictions to the beam line settings. In addition, the cooler is mounted in the tunnel of a synchrotron, the Main Injector, which magnetic fields move the electron beam by several $\mathrm{mm}$ in the time of ramping. This motion forces to keep the electron beam far from the crash scraper, decreasing its efficiency.

A dramatic reduction in the full discharge frequency came from an improvement of the protection system, when the time to close the gun was decreased from $1 \mathrm{~ms}$ down to $1 \mu \mathrm{s}$. The signal to close the gun is sent if the terminal voltage drops by more than $5 \mathrm{kV}$ (Fig. 2b), and neither the beam position nor the beam envelope change significantly. This improvement allows operating with no crash scrapers.

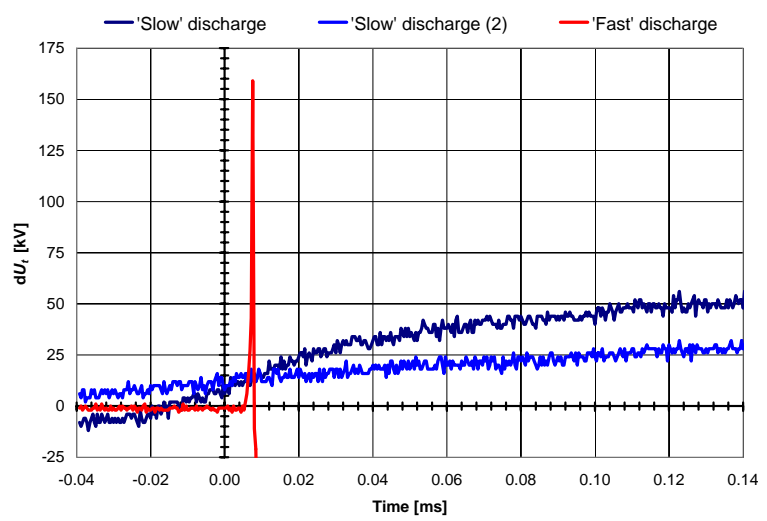

(a)

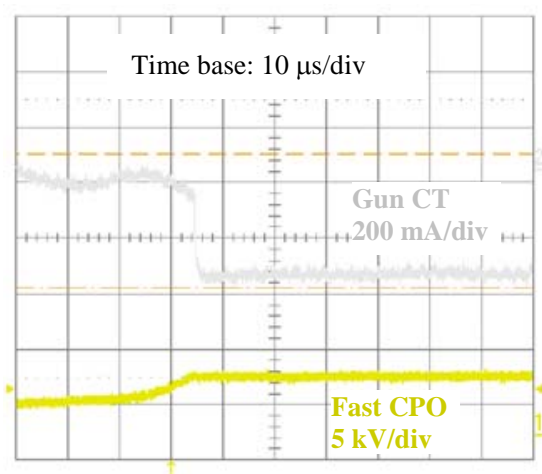

(b)

FIGURE 2. (a) Oscillograms of the terminal voltage in the time of a 'fast' (red) and 'slow' (blue and dark blue) full discharges. (b) Oscillograms of a recirculation interruption. The yellow trace is the terminal voltage, and the grey trace is the cathode current.

\section{Fast discharges}

Full discharges provoked by the primary beam are "slow", i.e. have a tens of $\mu$ long pre-history (Fig. 2a). The terminal is discharged first at the rate of 3- $300 \mathrm{~V} / \mu \mathrm{s}$, which corresponds to a current loss of $1-100 \mathrm{~mA}$, and only later, when the beam touches the tube electrodes, the main drop of voltage occurs. In contrary, all recently observed full discharges are "fast" (the red curve in Fig. 2a). The voltage derivative is large from the very beginning and corresponds to the current discharging the terminal. At $\sim 10 \mathrm{~A}$, this current is much larger than the beam current. It can only be interpreted as a developing discharge on the vacuum side of the deceleration tube. Typically, after several microseconds the derivative increases by orders of magnitude. It means that the vacuum discharge has already shorted a large portion of the tube, while the terminal potential is almost unchanged. As a result, the voltage between electrodes of the unaffected part reaches the threshold at which the spark gaps mounted at each 
electrode fire. From this point on, the terminal discharges through the gaps and the electric strength of the remaining part of the tube does not deteriorate.

Frequency of these discharges correlates with changes in the potential distribution along the tubes caused by secondary electrons escaping the collector. The redistribution of potential is estimated by measuring the currents of the tube resistive divider strings at the tube bottom and top (Fig. 3).

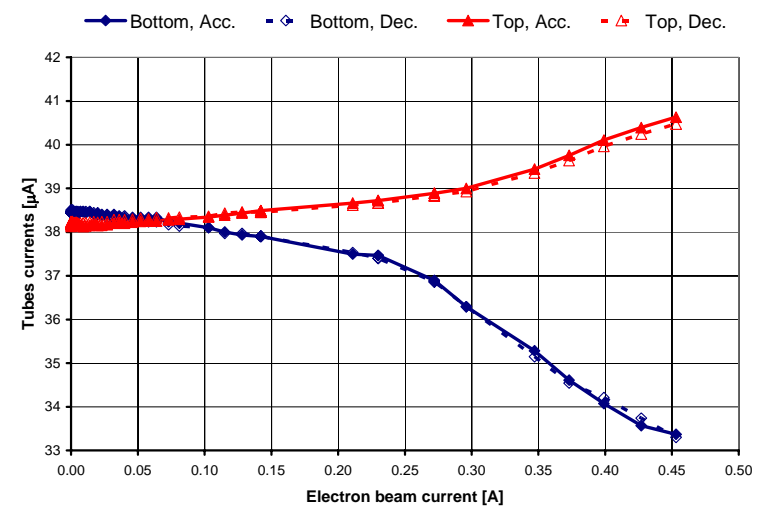

(a)

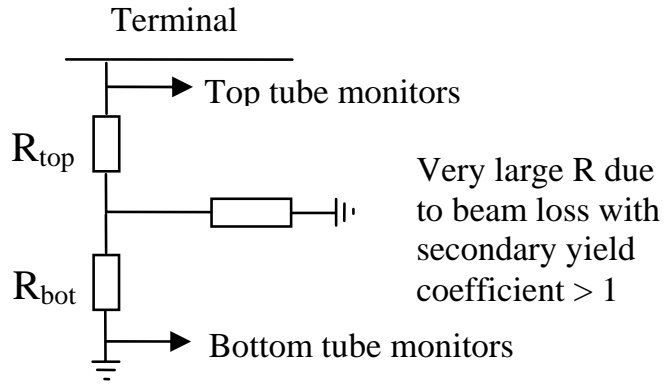

(b)

FIGURE 3. (a) Acceleration and deceleration DC tube monitor currents as functions of the electron beam current. (b) Simplified electrical schematic of the divider strings of the accelerating tubes assuming localized beam loss to the tubes.

The bottom current is always decreasing with the beam current, which means that the coefficient of secondary emission from the tube electrodes is above unity. Hence, the electric field between electrodes is increasing at the top portion of the tube and may eventually lead to a full discharge. Assuming electrons are lost to a single electrode, one can estimate from Fig. 3a the position of the electrode in the resistive string using the simple model of Fig. 3b. This position is found to be $1 / 3$ from the bottom and do not change with the beam current.

Though the losses occur low in the tube, the balance of currents still causes the maximum overvoltage to appear at the top of the tube. Moreover, another indication that full discharges originate from the top sections is that the largest degradation of the tube's electric strength is always observed in the first two top sections of the deceleration tube, while its lowest sections and the acceleration tube do not deteriorate.

This model of a direct relation between the discharge frequency and the tube overvoltage gives several guidelines that have proven to be useful:

- $\quad$ keep the tube strength well above the nominal electric field;

- minimize the amount of the current lost at the tube rather than the total loss;

- move the location of the loss as close to the tube bottom as possible.

On the other hand, the model can't numerically predict the tolerable level of the resistive string current change. At $I_{b}=0.45$ A, Fig. 3a predicts an overvoltage of $6.5 \%$. Attempts to stay an hour at this current resulted in full discharges, while the Pelletron stayed reliably with no beam with the terminal voltage increased by the same $6.5 \%$. Also, in Fig. 3a the currents recorded on the acceleration and deceleration sides 
coincide. Because the resistive strings are electrically connected at 5 intermediate voltage levels, the equality of currents indicates that the loss occurs in the middle portion of the tube. Therefore, the overvoltage is identical on both sides, but only the deceleration tube is affected by the discharges. One can speculate that while the frequency of the discharges is determined by overvoltage, they are triggered by secondary particles created by the lost electrons.

Note that recently, we did not observe full discharges while operating at $I_{b}=0.1$ 0.2 A. However, attempts to work at $0.4-0.6$ A still resulted in full discharges, and after $\sim 10$ discharges we had to repeat conditioning of the top sections ( $\sim 6$ hours).

\section{SUMMARY}

Decreasing the frequency of the full discharges was an important prerequisite for the electron cooling of $8 \mathrm{GeV}$ antiprotons in the Recycler ring. The decrease was achieved by careful adjustment of the beam envelope in the acceleration tube, by implementation of a fast gun shut-off system, and by working at settings where changes of the resistive strings currents are below $1 \mu \mathrm{A}$. Presently, the operation at the beam currents of $0.1-0.2$ A does not produce any full discharges in weeks.

\section{ACKNOWLEDGMENTS}

The system of the fast gun shut-off was developed by G. Saewert with contributions by J. Crisp and support from J. Simmons. Commissioning and operation of the cooler was performed by the entire ECool group. Authors acknowledge participating in the data taking and analysis presented in this report by our summer student D. Artamonov. Finally, we greatly appreciate the help provided by M. Tiunov and D. Myakishev for the SAM/SuperSAM/BEAM codes.

\section{REFERENCES}

1. S. Nagaitsev, these proceedings

2. A. Shemyakin, et al., these proceedings

3. Pelletrons are manufactured by the National Electrostatics Corporation, www.pelletron.com

4. A. Burov, et al., in: Peter Lucas, Sara Weber (Eds.), Proceedings of the 2001 Particle Accelerator Conference, Chicago, 2001, IEEE, Piscataway, NJ, 2001, p. 2548.

5. A. Shemyakin, et al., Nucl. Instr. and Meth. A, 532 (2004) 403-407

6. B. Fomel, M. Tiunov, V. Yakovlev. SAM - an interactive code for evaluation of electron guns, Preprint Budker INP 96-11, 1996

7. D.G. Myakishev, M.A. Tiunov, V.P.Yakovlev, SUPERSAM - the Code for Calculation of High-Perveance Guns, Program and Abstracts of 6th International Computational Accelerator Physics Conference, Germany, 2000

8. M. Tiunov, BEAM - 2D Code For Simulation Of High Perveance Beam Dynamics In Long Systems, Proceedings of SCHEF'99, Dubna, Russia, 1999, pp. 202-208 